\title{
MORFOLOGIA E ESTABILIDADE DE AGREGADOS SUPERFICIAIS DE UM ARGISSOLO VERMELHO- AMARELO SOB DIFERENTES MANEJOS DE LONGA DURAÇÃO E MATA ATLÂNTICA SECUNDÁRIA ${ }^{(1)}$
}

\author{
Clério Hickmann ${ }^{(2)}$, Liovando Marciano da Costa ${ }^{(3)}$, Carlos Ernesto \\ G.R. Schaefer ${ }^{(3)}$ \& Raphael Bragança Alves Fernandes ${ }^{(3)}$
}

\begin{abstract}
RESUMO
Experimentos de longa duração submetidos a diferentes manejos de solo fornecem importantes informações quando as alterações nas propriedades físicas, químicas e biológicas são avaliadas e comparadas às das condições originais do solo. Este estudo teve como objetivo avaliar a morfologia externa e a estabilidade de agregados superficiais de um Argissolo Vermelho-Amarelo sob diferentes tipos de manejo em experimento de longa duração e nas condições sob Mata Atlântica secundária. Foram coletadas amostras de solo deformadas e indeformadas na camada de 0-5 cm, em dois tipos de manejo de solo: plantio direto (PD) e preparo com arado de disco + grade pesada (AD + GP). Também foi avaliada, como referência do estado de agregação original do solo, uma área de Mata Atlântica secundária (MS) adjacente ao experimento. As amostras indeformadas foram impregnadas com resina de poliéster e, na sequência, lâminas delgadas com $30 \mu \mathrm{m}$ de espessura foram confeccionadas. Posteriormente, as lâminas foram examinadas em microscópio óptico, acoplado à câmera digital, para obtenção de imagens fotográficas mediante seleção aleatória de campos nas lâminas. As imagens geradas foram processadas pelo programa de computador Adobe Photoshop CS3 para análise de parâmetros relacionados à morfologia externa de agregados (perímetro, área, comprimento do maior eixo, comprimento do menor eixo, diâmetro de Feret, alongamento e arredondamento), que visam fornecer informações sobre o tamanho,
\end{abstract}

(1) Parte da Dissertação de Mestrado do primeiro autor apresentada ao Curso de Pós-Graduação em Solos e Nutrição de Plantas, Universidade Federal de Viçosa - UFV. Apoio financeiro FAPEMIG. Recebido para publicação em 16 de setembro de 2010 e aprovado em 24 de junho de 2011.

(2) Doutorando em Ciência do Solo da Universidade Federal de Lavras - UFLa. Campus Universitário, CEP $37200-000$ Lavras (MG). Bolsista FAPEMIG. E-mail: clerioh@gmail.com

(3) Professor do Departamento de Solos, Universidade Federal de Viçosa - UFV. Av. P. H. Rolfs s/n, CEP 36570-000 Viçosa (MG). Bolsista do CNPq. E-mails: liovandomc@yahoo.com.br; carlos.schaefer@ufv.br; raphael@ufv.br 
a forma e a rugosidade dos agregados. Para as amostras deformadas, além da estabilidade dos agregados em água em classes de tamanho 4-2; 2-1; 1-0, 5; 0,5-0,25; 0,25-0,105; e < 0,105 mm de diâmetro, foi determinado o C orgânico total (COT). Foram calculados: diâmetro médio ponderado (DMP), diâmetro médio geométrico (DMG) e índice de estabilidade de agregados (IEA). Houve predomínio da classe de agregados de 4-2 mm nos dois tipos de manejo e na MS, sendo superior no solo sob MS e PD. Os índices de agregação DMG, DMP e IEA foram semelhantes no PD e na MS, sendo maiores que o do manejo por AD + GP. O PD promoveu diferenças morfológicas nos agregados em comparação ao AD + GP, mostrando proximidade com a referência MS.

Termos de indexação: agregação, semeadura direta, preparo dolo.

\title{
SUMMARY: MORPHOLOGY AND STABILITY OF SOIL AGGREGATES IN A RED YELLOW ARGISOL UNDER DIFFERENT MANAGEMENT LONG-TERM AND SECONDARY ATLANTIC FOREST
}

\begin{abstract}
Long-term experiments testing different soil managements provide valuable information in the comparison of alterations in physical, chemical and biological soil properties with soil under natural vegetation. This long-term study was carried out to evaluate the external morphology and the stability of aggregates in the surface layer of a Yellowish-Red Argisol under different managements for comparison with secondary Atlantic Forest. Disturbed and undisturbed soil samples were collected from the 0-5 cm layer from soil under no-tillage $(P D)$ and disk plow + disk harrow $(A D+G P)$, as well as from a secondary Atlantic forest (MS) adjacent to the experimental area as a reference of the original soil aggregation. The undisturbed samples were resin-impregnated and cut in $30 \mu \mathrm{m}$ thick slides, which were observed under an optical microscope with a digital camera. Images were taken from random sites of the thin sections and processed using Adobe Photoshop CS3 to measure parameters related to the external morphology of the aggregates, as follows: perimeter, area, the longest and shortest axis, Ferret diameter, elongation and roundness. These variables provide information about the aggregate size, shape and rugosity. Besides the aggregate stability in water, in the size classes - 4-2; 2-1; 1-0.5; 0.5-0.25; 0.25-0.105 and diameter $<0.105 \mathrm{~mm}$, the total organic carbon (COT) was determined for the disturbed soil samples. The weighted and geometric mean diameters (DMP and DMG) as well as the aggregate stability index (IEA) were calculated. The 4-2 $\mathrm{mm}$ aggregate class predominated in both treatments and MS, but was higher in MS and PD soils. The DMG, DMP and IEA were similar in PD and MS, but higher than under $A D+G P$ management. The no-tillage treatment $(P D)$ resulted in morphological changes in the aggregates, compared to the $A D+G P$, tending to approach the properties of soil under $M S$.
\end{abstract}

Index terms: aggregation, no-tillage, tillage.

\section{INTRODUÇÃO}

O uso intensivo do solo, aliado a condições inadequadas de manejo, promove a deterioração das propriedades físicas, devido principalmente às modificações na estrutura original, o que favorece a formação de agregados pouco estáveis no solo (Castro Filho et al., 1998). A magnitude com que as alterações ocorrem depende do tipo de solo e dos sistemas de manejo utilizados. O efeito mais prejudicial ocorre em camadas superficiais expostas, em tipos de manejo que adotam revolvimento intensivo do solo, visto que o manejo inadequado de preparo do solo por máquinas e implementos agrícolas leva à formação de uma camada superficial compactada (Argenton et al., 2005) e à redução do teor de matéria orgânica, principal agente de formação e estabilização dos agregados (Wendling et al., 2005), sendo ambos os processos apontados como as principais causas da degradação estrutural do solo. Do ponto de vista agrícola, a estrutura do solo é um dos atributos mais importantes, pois está relacionado com as disponibilidades de ar e água às raízes das plantas, 0 suprimento de nutrientes, a resistência mecânica à penetração e com o desenvolvimento do sistema radicular (Corrêa, 2002). Portanto, a manutenção de um adequado estado de agregação do solo é condição primordial para garantir alta produtividade agrícola. 
A utilização de sistemas conservacionistas tem se tornado efetiva estratégia para reverter o quadro da degradação das condições físicas, químicas e biológicas do solo, causadas por manejos que demandam revolvimento. Entre os sistemas de preparo do solo, o plantio direto é o conservacionista, uma vez que, ao contrário dos tipos de manejo que envolvem aração e gradagens, este interfere muito pouco na estrutura do solo, preservando ao máximo os resíduos culturais, tornando-se efetiva estratégia, principalmente, para aumento do estoque de C no solo (Kern \& Johnson, 1993; Corazza et al., 1999; Costa et al., 2008) e estabilidade de agregados (Castro Filho et al., 1998; Wendling et al., 2005).

A importância da agregação do solo foi alvo de estudos quanto à morfologia dos agregados. Dadalto et al. (1989) concluíram que os agregados mostram diferenças quanto a tamanho, forma, estabilidade e adesão entre si e que alterações nas condições naturais do solo por sistemas de manejo podem alterar o tamanho, a distribuição e a estabilidade dos agregados. Olszevski et al. (2004) estudaram a morfologia de agregados por meio de análise de imagens de um Latossolo submetido a diferentes formas de preparo por quatro anos e concluíram que houve mudanças na morfologia dos agregados, relacionadas principalmente à área, comprimento, alongamento e arredondamento. Por sua vez, Melo et al. (2008) verificaram que a mineralogia teve efeitos significativos no tamanho e na morfologia externa de agregados em Latossolos distintos, quando também analisados por imagens.

Uma vez que as mudanças estruturais influem diretamente na morfologia dos agregados, considerase fundamental o uso de novos métodos de estudos morfológicos para permitir o acompanhamento das modificações desse atributo nos solos cultivados. Dessa forma, experimentos de longa duração fornecem importantes informações que podem servir como indicativo do tipo de manejo mais sustentável para minimizar os impactos causados no solo, quando comparados às condições originais de uso.

Este estudo teve como objetivo avaliar a morfologia externa e a estabilidade de agregados superficiais de um Argissolo Vermelho-Amarelo sob diferentes tipos de manejo em experimento de longa duração e sob Mata Atlântica secundária.

\section{MATERIAL E MÉTODOS}

O estudo foi realizado no ano agrícola de 2008, a partir de um experimento de campo conduzido na Estação Experimental da Universidade Federal de Viçosa, localizada no município de Coimbra, MG $\left(20^{\circ}\right.$ $45^{\prime} \mathrm{S}$ e $\left.42^{\circ} 51^{\prime} \mathrm{W}\right)$. O solo do experimento é um Argissolo Vermelho-Amarelo distrófico, de textura argilosa (Embrapa, 2006), que contém na camada de $0-5 \mathrm{~cm}$, após 23 anos de condução do experimento, os seguintes atributos químicos e físicos: $\mathrm{pH}\left(\mathrm{H}_{2} \mathrm{O}\right): 5,0$; $\mathrm{P}$ e K disponíveis (Mehlich-1): 19,6 e $147 \mathrm{mg} \mathrm{dm}^{-3}$, respectivamente; $\mathrm{Ca}^{2+}, \mathrm{Mg}^{2+} \mathrm{e} \mathrm{Al}^{3+}$ trocáveis $(\mathrm{KCl}$ $\left.1 \mathrm{~mol} \mathrm{~L}^{-1}\right): 1,9,0,7$ e $0,1 \mathrm{cmol}_{\mathrm{c}} \mathrm{dm}^{-3}$, respectivamente; saturação por bases de $36 \%$; e teores de areia grossa, areia fina, silte e argila de 346, 151, 81 e $422 \mathrm{~g} \mathrm{~kg}^{-1}$, respectivamente. A altitude, temperatura, precipitação pluvial e umidade relativa do ar médias anuais no local são: $700 \mathrm{~m}, 19^{\circ} \mathrm{C}, 1.400 \mathrm{~mm}$ e $85 \%$, respectivamente.

A área experimental originalmente sob Floresta Atlântica foi desmatada no início da década de 1930 e cultivada anualmente, durante 54 anos, com as culturas de milho e feijão com preparo convencional (mediante aração com arado de discos, seguida de duas gradagens com grade leve niveladora). Em 1985 foi realizada a adequação da área, visando à implantação dos tipos de manejos a serem estudados. A adequação constou da correção da acidez do solo com aplicação de $4,0 \mathrm{Mg} \mathrm{ha}^{-1}$ de calcário dolomítico e da fertilidade do solo, com aplicações anuais diferenciadas na forma de NPK (sulfato de amônio, superfosfato simples e cloreto de potássio), conforme as exigências nutricionais das culturas estudadas ao longo dos anos de condução do experimento. Durante o período experimental a área foi submetida, em média, a um preparo de solo por ano para o cultivo das culturas de milho, feijão, soja e trigo. Realizaram-se cultivos anuais, procedendo-se à semeadura e colheita entre os meses de outubro e março, enquanto nos demais meses do ano a área permanecia em pousio, exceto no período de 1996 a 1998 e nos anos de 2001, 2003 e 2008, quando também foram realizados cultivos entre os meses de janeiro e março.

$\mathrm{O}$ experimento foi realizado em delineamento em blocos ao acaso, com dois tratamentos e quatro repetições, mais um tratamento adicional (testemunha). Os tratamentos foram: plantio direto, com solo revolvido em forma de sulcos somente nas linhas de semeadura, utilizando semeadora de tração motorizada (PD) e revolvimento do solo com arado de disco + grade pesada, com única gradagem para efetuar a trituração dos restos culturais, seguido por única aração com arado de discos fixos com tração motorizada (AD + GP). Como referência (testemunha), foi demarcada uma área de Mata Atlântica secundária adjacente ao experimento, com tamanho de parcela, tipo de solo (Argissolo Vermelho-Amarelo) e repetições equivalentes às formas de preparo do solo.

$\mathrm{Na}$ avaliação da estabilidade de agregados, foram coletadas na camada de $0-5 \mathrm{~cm}$, mediante auxílio de pá-de-corte, cinco amostras simples para formarem uma amostra composta, nas quatro repetições de cada tratamento. Foram selecionados, com o devido cuidado, torrões para evitar a desagregação induzida. Posteriormente, as amostras foram pré-selecionadas, secas ao ar, acondicionadas e agitadas em jogo de peneiras sobrepostas, tendo as peneiras superior e inferior malhas de 4 e $2 \mathrm{~mm}$, respectivamente. Amostras de $25 \mathrm{~g}$ de grânulos foram submetidas ao umedecimento lento com auxílio de atomizador 
manual, por $2 \mathrm{~h}$. A seguir, as amostras foram colocadas em um conjunto de peneiras com malhas de $2 ; 1 ; 0,50 ; 0,25$; e $0,105 \mathrm{~mm}$ de diâmetro, imersas em água e agitadas por um oscilador mecânico de Yoder (Kemper \& Chepil, 1965), de frequência igual a 38 ciclos $\mathrm{min}^{-1}$ e $3,5 \mathrm{~cm}$ de amplitude vertical, durante $15 \mathrm{~min}$, para obtenção de proporções de classes de agregados de tamanhos $4-2 ; 2-1 ; 1-0,5 ; 0,5-0,25$; 0,25-0,105; e < 0,105 mm de diâmetro.

Foram calculados: diâmetro médio ponderado (DMP), por meio da equação $\mathrm{DMP}=\Sigma_{\mathrm{i}=1}^{\mathrm{n}}$ (xi.wi), em que wi= proporção (\%) de cada classe de agregados em relação ao total de classes e xi $=$ diâmetro médio das classes (mm); diâmetro médio geométrico (DMG), a partir da equação $\mathrm{DMG}=\operatorname{antilog} \Sigma($ wi $\log \mathrm{xi}) / \Sigma($ wi); $\mathrm{e}$ índice de estabilidade de agregados (IEA), por meio da equação IEA= (Ps-wp0,105 - areia)/(Ps-areia)100, em que Ps é a massa da amostra seca (g) e wp0,105 é a massa dos agregados da classe $<0,105 \mathrm{~mm}$ (g). Para determinação do C orgânico total (COT), procedeu-se à mesma forma de amostragem descrita na análise de agregados. As amostras, depois de homogeneizadas e secas ao ar, foram analisadas conforme método proposto por Yeomans \& Bremner (1988).

$\mathrm{Na}$ análise morfológica dos agregados, foram coletadas duas amostras indeformadas por tratamento. As amostras foram coletadas por meio de anéis com volume aproximado de $100 \mathrm{~cm}^{3}$. Elas foram secas em estufa de circulação de ar forçada e, posteriormente, em câmara fechada com sílica-gel e vapor de acetona, sendo então impregnadas com resina de poliéster, segundo método descrito por Castro (1985) e Murphy (1986). Após terem passado pelo processo de cura da resina, as amostras foram cortadas com auxílio de serra de disco diamantado, para proceder-se à confecção de lâminas delgadas de $30 \mu \mathrm{m}$ de espessura. As lâminas foram fotografadas em microscópio óptico Olympus CX-31 com máquina digital acoplada, com aumento de 40 vezes. Foram selecionados aleatoriamente 20 agregados por lâmina, em vários campos examinados (Figura 1). Após obtenção das imagens digitais, estas foram processadas e medidas com a utilização do programa Adobe Photoshop CS3. A resolução da medida foi determinada pelo tamanho do pixel usado na digitalização da imagem. Foram medidos os parâmetros: perímetro $(\mathrm{Pm})$ - corresponde ao comprimento da projeção do limite exterior do agregado; comprimento do menor eixo (CME) - comprimento de uma linha traçada perpendicularmente ao maior eixo do agregado; comprimento do maior eixo (CMA) - comprimento de uma linha traçada na maior distância no agregado; área do agregado (Ar) - calculada a partir da fórmula Ar: $\pi$ (CMA.CME); alongamento (Al) - pela relação entre CME e CMA; arredondamento (Ard) - calculado a partir da fórmula $\mathrm{Ar}=(4 \pi \mathrm{Ar}) / \mathrm{Pm}^{2}$; e diâmetro de Feret (DF) - calculado a partir da fórmula $\mathrm{DF}=\sqrt{4 \mathrm{Ar} / \pi}$. As lâminas também foram escaneadas mediante utilização de um scanner HP 6100C, com capacidade de resolução de

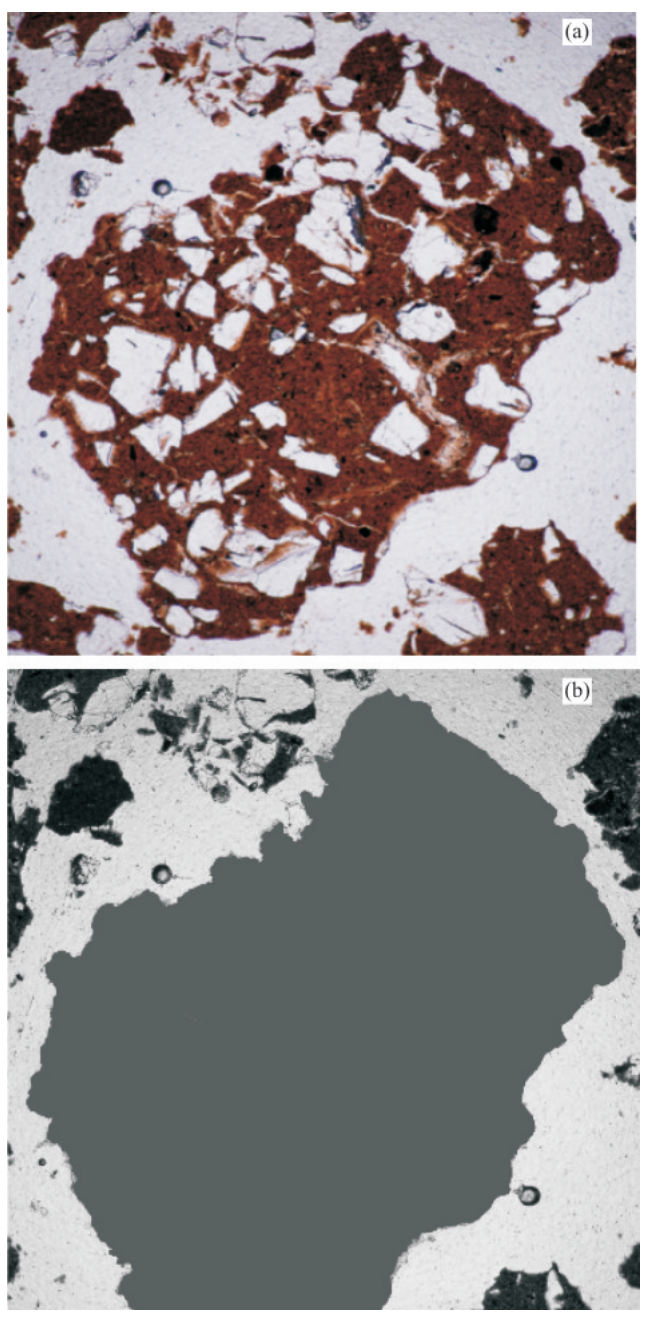

Figura 1. Microfotografia de agregado selecionado na lâmina (a) e representação da área de estudo (b). Escala: $1 \mathrm{~mm}$.

2.400 dpi, para obtenção da distribuição das características morfométricas da porosidade.

Os resultados foram submetidos à análise de variância, considerando como fontes de variação os diferentes tipos de manejo de solo e a Mata Atlântica secundária. As médias foram comparadas pelo teste de Tukey a $5 \%$. Utilizou-se o sistema computacional WinStat (Machado \& Conceição, 2002) como auxílio no procedimento estatístico.

\section{RESULTADOS E DISCUSSÃO}

\section{Distribuição dos agregados em classes de tama- nho}

Na distribuição dos agregados estáveis em água, ocorreu predomínio da classe de tamanho de 4-2 mm, 
representando 87,2, 78,4 e 48,5\% na MS, PD e $\mathrm{AD}+\mathrm{GP}$, respectivamente, sendo superior $(\mathrm{p}<0,05)$ na MS e no PD (Quadro 1). A ampla superioridade dessa classe de agregados influenciou nos elevados valores observados para os índices diâmetro médio geométrico (DMG) e diâmetro médio ponderado (DMP), sendo considerados superiores aos encontrados comumente na literatura (Castro Filho et al., 1998; Wendling et al., 2005; Matos et al., 2008) (Quadro 2). O índice DMP será maior quanto maior for a proporção da classe de agregados de maior tamanho, ao passo que o índice DMG representa apenas uma estimativa das classes de agregados de maior ocorrência. Já o elevado IEA deve-se às baixas quantidades de agregados não estáveis em água, tanto nos solos não revolvidos (MS e PD) como no preparo convencional $(\mathrm{AD}+\mathrm{GP})$, pois esse índice representa apenas a medida de agregação total do solo, desprezando a proporção pertencente à classe $<0,105 \mathrm{~mm}$, que é contabilizada pela diferença do somatório das outras classes.

Durante os 23 anos de condução de experimentos na área, aumentou quase $30 \%$ a proporção da classe de tamanho de 4-2 mm no manejo PD, em relação ao preparo AD + GP (Quadro 1). Esses resultados corroboram os obtidos por Salton et al. (2008) e Vezzani et al. (2011), que verificaram incremento da mesma classe de agregados no manejo PD em Latossolo e Argissolo Vermelhos, respectivamente, ao comparálo com o preparo convencional em experimentos de longa duração.

Observou-se que o manejo PD favoreceu o incremento em 75,4, 39,6 e 4,7 \% do DMG, DMP e IEA, respectivamente, em relação ao preparo com $\mathrm{AD}+\mathrm{GP}$. Essa superioridade nos valores indicativos da agregação pode ser atribuída ao maior acúmulo de C orgânico total (COT) no manejo PD (Quadro 2). O COT foi maior $(\mathrm{p}<0,05)$ no solo da MS, sendo seguido pelo $\mathrm{PD}$, e menor no AD + GP. Em experimentos de longa duração conduzidos em diferentes regiões do Brasil, têm sido comprovados os efeitos do acúmulo de resíduos vegetais, sobretudo na superfície do solo em sistema plantio direto, formando consequentemente ca- madas com teores com gradientes diferenciáveis e maiores de C orgânico (Castro Filho et al., 1998; Corazza et al., 1999; Bertol et al., 2004; Argenton et al., 2005; Costa et al., 2008). Em situações semelhantes, estudos conduzidos por Castro Filho et al. (1998) e Wendling et al. (2005) também verificaram correlações positivas do COT com índices de agregação.

A relação entre agregados do solo e o C do solo já foi alvo de muitos estudos. Six et al. (2004), em revisão sobre processos de formação e organização de agregados, relatam que Emerson (1959) considerou a formação de microagregados em função da ligação entre domínios de argila orientadas com a matéria orgânica do solo (MOS) e partículas de quartzo, sendo a MOS proporcional à área superficial dos domínios. Citam também o conceito hierárquico de agregação proposto por Tisdall \& Oades (1982), os quais postulam que partículas primárias livres e agregados de tamanho do silte são unidos por agentes ligantes persistentes, como matéria orgânica humificada ou complexos com cátions polivalentes, óxidos e aluminossilicatos, formando microagregados (20 a $250 \mu \mathrm{m})$. Esses

Quadro 2. Diâmetro médio geométrico (DMG), diâmetro médio ponderado (DMP), índice de estabilidade de agregados (IEA) e teor de carbono orgânico total (COT) na camada de 0$5 \mathrm{~cm}$ de um Argissolo Vermelho-Amarelo sob diferentes tipos de manejo, após 23 anos de instalação, e sob Mata Atlântica secundária

\begin{tabular}{lcccc} 
Tipo de manejo $^{(1)}$ & DMG & DMP & IEA & COT \\
\hline & & & & \\
\cline { 2 - 3 } MS & $2,35 \mathrm{a}$ & $2,73 \mathrm{a}$ & $96,1 \mathrm{a}$ & $3,14 \mathrm{a}$ \\
$\mathrm{PD}$ & $2,14 \mathrm{a}$ & $2,61 \mathrm{a}$ & $96,0 \mathrm{a}$ & $2,17 \mathrm{~b}$ \\
AD + GP & $1,22 \mathrm{~b}$ & $1,87 \mathrm{~b}$ & $91,7 \mathrm{~b}$ & $1,59 \mathrm{c}$ \\
CV (\%) & 11,4 & 7,9 & 1,4 & 4,6 \\
\hline
\end{tabular}

(1) MS: Mata Atlântica secundária; PD: plantio direto; AD + GP: arado disco + grade pesada. CV: coeficiente de variação. Médias seguidas da mesma letra na coluna não diferem entre si pelo teste de Tukey $(p<0,05)$.

Quadro 1. Distribuição das classes de tamanhos dos agregados estáveis em água na camada de 0-5 cm em um Argissolo Vermelho-Amarelo sob diferentes tipos de manejo, após 23 anos de instalação, e sob Mata Atlântica secundária

Tipo de manejo $^{(1)} \quad 4-2 \mathrm{~mm} \quad 2-1 \mathrm{~mm} \quad 1-0,5 \mathrm{~mm} \quad 0,5-0,25 \mathrm{~mm} \quad 0,25-0,105 \mathrm{~mm} \quad<0,105 \mathrm{~mm}$

\begin{tabular}{|c|c|c|c|c|c|c|}
\hline & & & & $\%$ & & 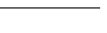 \\
\hline MS & $87,22 \mathrm{a}$ & $6,73 \mathrm{~b}$ & $0,99 \mathrm{~b}$ & $0,79 \mathrm{~b}$ & $0,50 \mathrm{~b}$ & $3,77 \mathrm{a}$ \\
\hline PD & $78,38 \mathrm{a}$ & $8,93 \mathrm{~b}$ & $4,09 \mathrm{~b}$ & $2,82 \mathrm{~b}$ & $1,69 \mathrm{~b}$ & $4,08 \mathrm{a}$ \\
\hline $\mathrm{AD}+\mathrm{GP}$ & $48,56 \mathrm{~b}$ & $19,13 \mathrm{a}$ & $10,60 \mathrm{a}$ & $8,54 \mathrm{a}$ & $5,22 \mathrm{a}$ & $7,94 \mathrm{a}$ \\
\hline $\mathrm{CV}(\%)$ & 13,4 & 29,8 & 55,1 & 42,9 & 48,4 & 40,6 \\
\hline
\end{tabular}

(1) MS: Mata Atlântica secundária; PD: plantio direto; AD + GP: arado disco + grade pesada. CV: coeficiente de variação. Médias seguidas da mesma letra na coluna não diferem entre si pelo teste de Tukey $(p<0,05)$. 
microagregados estáveis são unidos por agentes ligantes temporários (raízes ou hifas de fungos) e transientes (polissacarídeos derivados de microrganismos ou plantas), resultando em macroagregados $(>250 \mu \mathrm{m})$. Dessa forma, os microagregados foram classificados como mais estáveis e menos suscetíveis às alterações induzidas por práticas de manejo do solo do que os macroagregados. Logo em seguida, mencionam que Oades (1984) acrescenta uma alteração ao modelo hierárquico, na qual propõe que as raízes e hifas atuam como núcleos de formação de microagregados e, por serem ligantes temporários, ao se decomporem, formam fragmentos recobertos por mucilagens e incrustados de argilas, dando origem a novos agregados.

\section{Morfologia dos agregados}

De maneira geral, os parâmetros área (Ar), perímetro $(\mathrm{Pm})$, diâmetro de Feret (DM), alongamento (Al) e arredondamento (Ard) foram maiores $(\mathrm{p}<0,05)$ no solo da MS. Entretanto, não houve diferença $(p<0,05)$ entre os valores de comprimento do maior eixo (CMA) e menor eixo (CME) quando se comparou o solo da MS com os manejos PD e AD + GP (Quadro 3). Para todos os parâmetros, o PD mostrou tendência de maiores valores em relação ao $\mathrm{AD}+\mathrm{GP}$, concordando com Olszevski et al. (2004), que obtiveram resultados semelhantes ao avaliarem macroagregados em diferentes tipos de manejo em um Latossolo Vermelho. Esses resultados são consistentes com o fato de o sistema PD favorecer menores danos à estrutura do solo, em comparação ao preparo com arados e grades, em que há maior revolvimento e, consequentemente, quebra e pulverização dos agregados.

Os maiores valores de Ar no solo da MS são consistentes ao compará-los com a sua agregação (Quadro 1), uma vez que esse solo mostrou predomínio de agregados que favorecem maior área do agregado e comprimento do maior eixo (agregados maiores que $2 \mathrm{~mm}$ ), sendo este um dos componentes para calcular o Ar. Ressalta-se também que o parâmetro Ar foi favorecido no solo da MS, pelo fato de este representar um sistema que não sofreu alterações que permitem a fragmentação e quebra dos agregados, da forma como aconteceu com o preparo $\mathrm{AD}+\mathrm{GP}$ e, em menor nível, no manejo PD.

Verifica-se que os tipos de manejo que não promovem revolvimento do solo (PD e MS) apresentam maiores valores de $\mathrm{Pm}$, o que pode ser devido à maior proporção de agregados de maior tamanho e maior rugosidade externa. Entretanto, com o parâmetro Al, ou seja, estruturas com maior diferença entre o maior e o menor eixo (valores de alongamento mais próximos de 1,0 indicam agregados mais esféricos), foram encontrados menores valores no PD. Supõe-se que tipos de manejo que promovem menor revolvimento do solo devem mostrar menores valores de $\mathrm{Al}$, em razão da menor quebra de agregados, induzindo o crescimento da estrutura preferencialmente em uma direção. Com o parâmetro Ard, os agregados dos dois tipos de manejo e da MS mostraram rugosidade (valores de arredondamento menores que 1,0). Logo, maiores valores de Ard deverão ser encontrados nos agregados provenientes de tipos de manejo mais destrutivos à estrutura, pois estes tenderão a perder a rugosidade da superfície externa, diminuindo o valor do perímetro (comprimento da projeção do limite exterior do agregado) e tendendo à circularidade (Olszevski et al., 2004).

Melo et al. (2008), estudando a micromorfologia de agregados de classes diferentes, em Latossolos Bruno e Vermelho-Amarelo, concluíram que há efeito da qualidade da argila na morfologia externa e tamanho dos agregados. Segundo esses autores, para um determinado atributo morfológico (área, perímetro, arredondamento, diâmetro de Feret e compacidade), o efeito do teor e da qualidade dos minerais da fração argila em somente algumas classes de agregados pode ser atribuído à influência de outros fatores importantes na formação e definição dos agregados do solo. Esses fatores teriam relação com o ambiente, o manejo do solo, a influência da planta e as propriedades do solo, como textura, composição mineral, teor de C orgânico, processos pedogenéticos, atividade microbiana, íons trocáveis e umidade.

Quadro 3. Valores de perímetro (Pm), comprimento do maior eixo (CMA), comprimento do menor eixo (CME), diâmetro de Feret (DM), área (Ar), alongamento (Al) e arredondamento (Ard) de agregados na camada de 0-5 cm de um Argissolo Vermelho-Amarelo sob diferentes manejos, após 23 anos de instalação, e sob Mata Atlântica secundária

\begin{tabular}{|c|c|c|c|c|c|c|c|}
\hline Tipo de manejo ${ }^{(1)}$ & Pm & CMA & CME & DM & Ar & $\mathbf{A l}$ & Ard \\
\hline & & & & 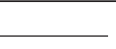 & $\mathrm{mm}^{2}$ & & \\
\hline MS & $18,41 \mathrm{a}$ & $2,86 \mathrm{a}$ & $1,77 \mathrm{a}$ & $5,79 \mathrm{a}$ & $26,96 \mathrm{a}$ & $0,95 \mathrm{a}$ & $0,98 \mathrm{a}$ \\
\hline $\mathrm{PD}$ & $14,04 \mathrm{~b}$ & $2,74 \mathrm{a}$ & $1,73 \mathrm{a}$ & $4,33 \mathrm{~b}$ & $15,79 \mathrm{~b}$ & $0,64 \mathrm{~b}$ & $0,94 \mathrm{~b}$ \\
\hline $\mathrm{AD}+\mathrm{GP}$ & $12,27 \mathrm{~b}$ & $2,24 \mathrm{a}$ & $1,34 \mathrm{a}$ & $3,83 \mathrm{~b}$ & $11,84 \mathrm{~b}$ & $0,89 \mathrm{a}$ & $0,96 \mathrm{ab}$ \\
\hline CV (\%) & 22,2 & 39,7 & 52,9 & 21,9 & 52,9 & 35,7 & 5,0 \\
\hline
\end{tabular}

(1) MS: Mata Atlântica secundária; PD: plantio direto; AD + GP: arado disco + grade pesada. CV: coeficiente de variação. Médias seguidas da mesma letra na coluna não diferem entre si pelo teste de Tukey $(p<0,05)$. 
A figura 2 representa a porosidade estrutural do solo da MS e dos manejos PD e AD + GP em escala macroscópica. Observou-se que o solo da MS mostrou uma rede de poros mais bem distribuídos (Figura 2a), o que provavelmente resultará em maior percolação de água nesta camada $(0-5 \mathrm{~cm})$. Contudo, o solo com PD (Figura 2b) assemelha-se muito ao da MS, em se tratando de conexão e distribuição dos poros. Esse efeito é explicado pela adequada estruturação do solo, resultante, principalmente, da maior agregação deste em relação ao preparo com $\mathrm{AD}+\mathrm{GP}(\mathrm{Quadros} 1 \mathrm{e} 2)$, visto que este (Figura 2c) mostrou predomínio de descontinuidade de poros, fato possivelmente ligado à desestruturação do solo causada pela fragmentaçãoe quebra dos agregados durante o revolvimento mediante uso de implementos agrícolas. Schaefer et al. (2001), ao estudarem a micromorfologia do mesmo solo, concluíram ser o tipo de manejo PD o que melhor mostra a distribuição e conexão entre os poros. Para esses autores, a superioridade da macroporosidade, associada à melhor estruturação do solo com PD, foi o fator determinante para favorecer a interconectividade entre os poros.

(a)

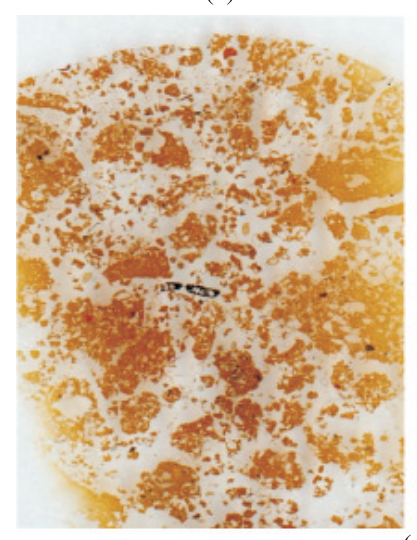

(c)

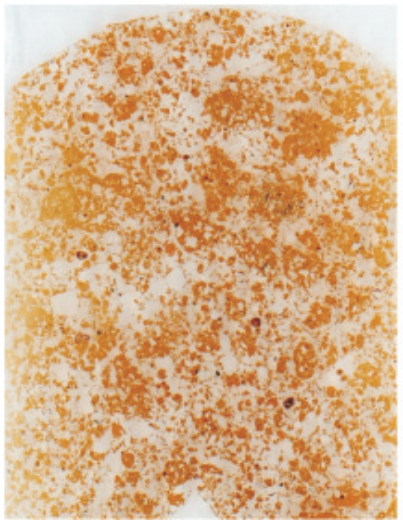

Figura 2. Fotografias das lâminas scaneadas, evidenciando a porosidade estrutural entre agregados na camada de $\mathbf{0}-\mathbf{5} \mathrm{cm}$ de um Argissolo Vermelho-Amarelo sob diferentes manejos, após 23 anos de instalação, e sob Mata Atlântica secundária. A: Mata Atlântica secundária (MS); B: plantio direto (PD); C: arado de disco + grade pesada $(A D+G P)$.

\section{CONCLUSÕES}

1. O cultivo do solo com plantio direto aumentou a estabilidade de agregados em água e os índices de agregação em superfície, em relação ao preparo convencional com arado de disco e grade pesada, sendo semelhante à Mata Atlântica secundária.

2. O manejo com plantio direto favorece a melhor distribuição e conexão entre poros e morfologia externa dos agregados na superfície, com maior aproximação das condições originais de estruturação do solo representado pela Mata Atlântica secundária.

\section{AGRADECIMENTOS}

À Fundação de Amparo à Pesquisa do Estado de Minas Gerais (FAPEMIG), pelo apoio financeiro na concessão da bolsa de estudo, e aos Departamentos de Solos e Fitotecnia da Universidade Federal de Viçosa (UFV), pela área de estudo cedida.

\section{LITERATURA CITADA}

ARGENTON, J.; ALBUQUERQUE, J.A.; BAYER, C. \& WILDNER, L.P. Comportamento de atributos relacionados com a forma da estrutura de um Latossolo Vermelho sob sistemas de preparo e plantas de cobertura. R. Bras. Ci. Solo, 29:425-435, 2005.

BERTOL, I.; ALBUQUERQUE, J.A.; LEITE, D.; AMARAL, A.J. \& ZOLDAN JUNIOR, W.A. Propriedades físicas do solo sob preparo convencional e semeadura direta em rotação e sucessão de culturas, comparadas às do campo nativo. R. Bras. Ci. Solo, 28:155-163, 2004.

CASTRO FILHO, C.; MUZILLI, O. \& PODANOSCHI, A.L. Estabilidade dos agregados e sua relação com o teor de carbono orgânico num Latossolo Roxo distrófico, em função de sistemas de plantio, rotações de culturas e métodos de preparo das amostras. R. Bras. Ci. Solo, 22:527538,1998

CASTRO, S.S. Impregnação de amostras de solo para confecção de lâminas delgadas. B. Inf. SBCS, 15:1-44, 1985.

CORAZZA, E.J.; SILVA, J.E.; RESCK, D.V.S. \& GOMES, A.C. Comportamento de diferentes sistemas de manejo como fonte ou depósito de carbono em relação à vegetação de cerrado. R. Bras. Ci. Solo, 23:425-432, 1999.

CORRÊA, J.C. Efeito de sistemas de cultivo na estabilidade de agregados de um Latossolo Vermelho-Amarelo em Querência, MT. Pesq. Agropec. Bras., 37:203-209, 2002.

COSTA, F.S.; BAYER, C.; ZANATTA, J.A. \& MIELNICZUK, J. Estoque de carbono orgânico no solo e emissões de dióxido de carbono influenciadas por sistemas de manejo no sul do Brasil. R. Bras. Ci. Solo, 32:323-332, 2008. 
DADALTO, G.G.; COSTA, L.M. \& MOURA FILHO, W. Alterações em características físicas de solos cultivados com pastagens. R. Ceres, 36:317-329, 1989.

EMERSON, W.W. The structure of soil crumbs. Eur. J. Soil Sci., 10:235-244, 1959.

EMPRESA BRASILEIRA DE PESQUISA AGROPECUÁRIA EMBRAPA. Centro Nacional de Pesquisa de Solos. Sistema brasileiro de classificação de solos. 2.ed. Brasília, Embrapa Produção de informação, 2006. 306p.

MELO, V.F.; MOURA, R.; TOLEDO, F.H.; LIMA, V.C. \& CHIDIN, A.A. Morfologia de agregados de Latossolos Bruno e Vermelho do estado do Paraná, avaliada por imagens obtidas em scanner. R. Bras. Ci. Solo, 32:85-99, 2008.

KEMPER, W.D. \& CHEPIL, W.S. Size distribution of aggregates. BLAKE, C.A.; EVANS, D.D.; WHITE, J.L.; ENSMINGER, L.E. \& CLARK, F.E., eds. Methods of soil analysis: Physical and mineralogical properties, including statistics of measurement and sampling. Madison, American Society of Agromony, 1965. p.499-510.

KERN, J.S. \& JOHNSON, M.G. Conservation tillage impacts on national soil and atmospheric carbon levels. Soil Sci. Soc. Am. J., 57:200-210, 1993.

MACHADO, A. \& CONCEIÇÃO, A.R. Programa estatístico WinStat Sistema de Análise Estatística para Windows. Versão 2.0. Pelotas, Universidade Federal de Pelotas, 2002.

MATOS, E.S.; SÁ MENDONÇA, E.; LEITE, L.F.C. \& GALVÃO. J.C.C. Estabilidade de agregados e distribuição de carbono e nutrientes em Argissolo sob adubação orgânica e mineral. Pesq. Agropec. Bras., 43:1221-1230, 2008.

MURPHY, C.P. Thin section preparation of soils and sediments. Berkhamsted, A. B. Academic Publishers, 1986.149p.

OADES, J.M. Soil organic-matter and structural stabilitymechanisms and implications for management. Plant Soil, 76:319-337, 1984.
OLSZEVSKI, N.; COSTA, L.M.; FERNANDES FILHO, E.I.; RUIZ, H.A.; ALVARENGA, R.C. \& CRUZ, J.C. Morfologia de agregados do solo avaliada por meio de análise de imagens. R. Bras. Ci. Solo, 28:901-909, 2004.

SALTON, J.C.; MIELNICZUK, J.; BAYER, C.; BOENI, M.; CONCEIÇÃO, P.C.; FABRÍCIO, A.C.; MACEDO, M.C.M. \& BROCH, D.L. Agregação e estabilidade de agregados do solo em sistemas agropecuários em Mato Grosso do Sul. R. Bras. Ci. Solo, 32:11-21, 2008.

SCHAEFER, C.E.G.R.; SOUZA, C.M.; VALLEJOS, F.J.M.; VIANA, J.H.M.; GALVÃO, J.C.C. \& RIBEIRO, L.M. Características da porosidade de um Argissolo VermelhoAmarelo submetido a diferentes sistemas de preparo de solo. R. Bras. Ci. Solo, 25:765-769, 2001.

SIX, J.; BOSSUYT, H.; DEGRYZE, S. \& DENEF, K. A history of research on the link between (micro)aggregates, soil biota, and soil organic matter dynamics. Soil Tillage Res., 79:7-31, 2004

TISDALL, J.M. \& OADES, J.M. Organic matter and water stable aggregates in soil. J. Soil Sci., 33:141-163, 1982.

VEZZANI, F.M. \& MIELNICZUK, J. Agregação e estoque de carbono em Argissolo submetido a diferentes práticas de manejo agrícola. R. Bras. Ci. Solo, 35:213-223, 2011.

WENDLING, B.; JUCKSCH, I.; MENDONÇA, E.S. \& NEVES, J.L.N. Carbono orgânico e estabilidade de agregados de um Latossolo Vermelho sob diferentes manejos. Pesq. Agropec. Bras., 40:487-494, 2005.

YEOMANS, J.C. \& BREMNER, J.M. A rapid and precise method for routine determination of organic carbon in soil. Comm. Soil Sci. Plant Anal., 19:1467-1476, 1988. 